\title{
Control of protein phosphorylation with a genetically encoded photocaged amino acid
}

\author{
Edward A Lemke ${ }^{1,3}$, Daniel Summerer ${ }^{1,3}$, Bernhard H Geierstanger ${ }^{2}$, Scott M Brittain ${ }^{2}$ \& Peter G Schultz ${ }^{1,2}$
}

We genetically encoded the photocaged amino acid 4,5-dimethoxy-2-nitrobenzylserine (DMNB-Ser, 1) in Saccharomyces cerevisiae in response to the amber nonsense codon TAG. This amino acid was converted to serine in living cells by irradiation with relatively low-energy blue light and was used to noninvasively photoactivate phosphorylation of the transcription factor Pho4, which controls the cellular response to inorganic phosphate ${ }^{1}$. When substituted at phosphoserine sites that control nuclear export of Pho4, 1 blocks phosphorylation and subsequent export by the receptor Msn5 (ref. 2). We triggered phosphorylation of individual serine residues with a visible laser pulse and monitored nuclear export of Pho4-GFP fusion constructs in real time. We observed distinct export kinetics for differentially phosphorylated Pho4 mutants, which demonstrates dynamic regulation of Pho4 function. This methodology should also facilitate the analysis of other cellular processes involving free serine residues, including catalysis, biomolecular recognition and ion transport.

Photocaged proteins can be used to noninvasively control the temporal and spatial activation of a variety of cellular processes (for reviews, see refs. 3-6). A number of approaches have been developed to directly photoactivate protein activity. These include the selective chemical $^{7}$ or enzymatic modification of specific amino acid side chains $^{8}$; alternatively, photocaged amino acids have been sitespecifically incorporated into proteins by in vitro transcription and translation methods ${ }^{9}$ or by chemical ligation of a truncated protein to a complementary synthetic photocaged peptide ${ }^{10}$. However, cellular studies using these methods are complicated because they typically require microinjection to deliver reagents into the cell ${ }^{11}$, or selective modification of a surface protein that is accessible to exogenous reagents ${ }^{12,13}$. More recently, it has been shown that photocaged amino acids can be genetically encoded in Escherichia coli and S. cerevisiae using orthogonal nonsense suppressor tRNA/aminoacyltRNA synthetase (aaRS) pairs (that is, pairs that do not cross-react with the endogenous tRNAs or aaRSs of the host cell $)^{14,15}$. This strategy offers the advantage that the modified proteins and peptides are generated inside the cell directly by the translational machinery and are genetically targeted to the desired site. Once established, such systems can be easily generalized to virtually any protein in the cell and allow photocontrol of protein function based on single amino acid mutations. However, so far only amino acids with the photolabile $o$-nitrobenzyl group ${ }^{14,15}$, which are photoactivated with UV light ( $<365 \mathrm{~nm}$, Supplementary Fig. 1 online), have been genetically encoded. The use of UV light restricts application of this technology for cellular studies because high-intensity UV light causes photoreactions in nucleic acids, destroys disulphide bonds and can cause other cellular damage. To overcome these limitations, we have genetically encoded 1 (Fig. 1a) in S. cerevisiae in response to the amber nonsense mutant TAG. In contrast to the $o$-nitrobenzyl group, the larger DMNB group has a higher quantum yield for photocleavage. More importantly, DMNB has a substantially red-shifted absorption spectrum, which allows photolysis of living cells with visible blue light from a standard laser scanning microscope (Supplementary Fig. 1) ${ }^{16}$. More
Figure 1 Incorporation of 1 into hSOD-33TAG and Pho4 phosphorylation scheme. (a) Structure of 1 , which was synthesized according to previous protocols ${ }^{29}$ (see also Supplementary Fig. 1). (b) GelCode blue-stained SDS polyacrylamide gel of hSOD-33TAG-His coexpressed with TRNA Leu LUA $_{\text {and }}$ and the LeuRS BH5 T252A mutant in the presence $(+1)$ or absence $(-1)$ of $4 \mathrm{mM} 1$ after Ni-NTA purification. $\mathrm{M}$, molecular weight marker in $\mathrm{kDa}$. (c) Pho4 can be phosphorylated by cyclin-CDK on five serine phosphorylation sites. S2 and S3 (amino acids 114 and 128 , respectively) are important for export and lie between the transcription activation domain and the nuclear localization sequence (NLS). There is no function yet assigned for the S1 site.
1Department of Chemistry, The Scripps Research Institute, 10550 North Torrey Pines Road SR202, La Jolla, California 92037, USA. ${ }^{2}$ Genomics Institute of the Novartis Research Foundation, 10675 John Jay Hopkins Drive, San Diego, California 92121-1125, USA. ${ }^{3}$ These authors contributed equally to this work. Correspondence should be addressed to P.G.S. (schultz@scripps.edu). 

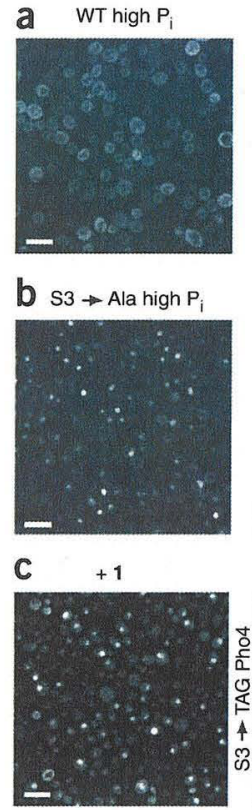

WT no $P_{i}$

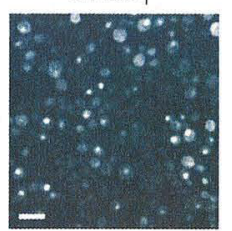

$\mathrm{S} 2 \rightarrow$ Ala high $\mathrm{P}$

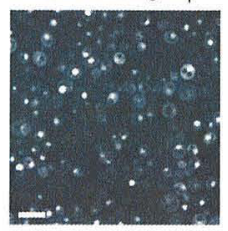
$-1$

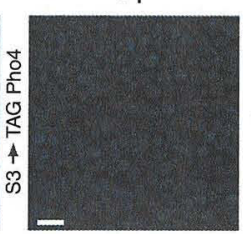

d Before photolysis

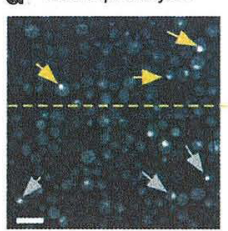

e

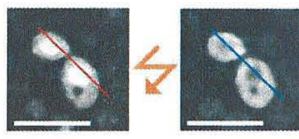

f Before photolysis

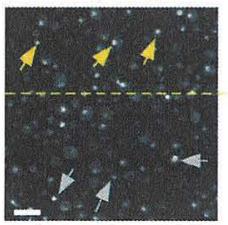

$140 \mathrm{~s}$ after photolysis
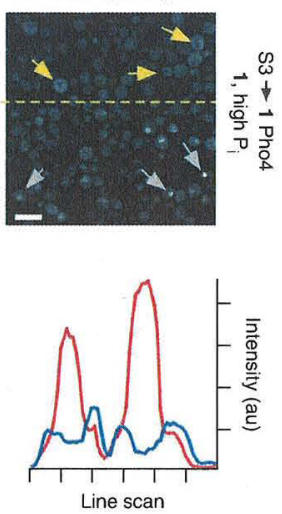

140 s after photolysis

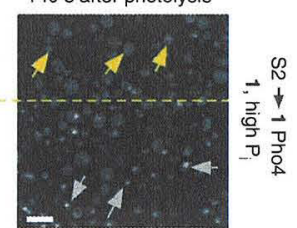

Figure 2 In vivo photolysis of the DMNB-Ser Pho4-GFP mutants. (a) Cytoplasmic localization of WT Pho4-GFP grown in high- $P_{i}$ medium and starved for phosphate. (b) S3 $\rightarrow$ Ala and S2 $\rightarrow$ Ala Pho4-GFP mutants localize to the nucleus in high $P_{i}$. (c) The S3 $\rightarrow$ TAG Pho4-GFP mutant localizes in the nucleus even in high $P_{i}$ medium if grown in the presence of 1 . (d) Fluorescent nuclei in the upper part (photolysed part, above yellow dashed line) of the image for the S3 $\rightarrow 1$ Pho4-GFP show a strong loss in fluorescence in the nucleus.

(e) Magnification of two cells before and after photolysis and corresponding line scan. (f) Photolysed S2 $\rightarrow \mathbf{1}$ Pho4-GFP mutant cells grown in high $\mathrm{P}_{i}$ show a strong loss of fluorescence originating from the nucleus. Signal amplification for images in $\mathbf{a}$ and $\mathbf{b}$ was reduced about three-fold to compensate for a $\sim$ seven-fold increased expression of protein. Scale bars are $10 \mu \mathrm{m}$.

yeast to grow under different concentrations of inorganic phosphate $\left(\mathrm{P}_{\mathrm{i}}\right)^{1,22}$. Under $\mathrm{P}_{\mathrm{i}^{-}}$ starved conditions, Pho4 is hypophosphorylated and localized in the nucleus, where it activates transcription of phosphate-respon-

over, no photocaged serine derivatives have been genetically encoded so far, and given that serine is critical to the function of many proteins-for example, as a catalytic residue, hydrogen bonding partner or site of post-translational modification-1 should have broad applicability in cellular and biochemical studies.

We used a previously reported orthogonal $E$. coli amber suppressor tRNA/aaRS (tRNA Leu5/LeuRS) ${ }^{15}$ to incorporate 1 into proteins in yeast and generated a library of $\sim 10^{7}$ LeuRS mutants in which residues Met40, Leu41, Tyr499, Tyr527 and His537 in the leucine binding site were randomized (see ref. 17 for X-ray structure). Next, we used a selection scheme to isolate a LeuRS mutant that selectively uses 1 . The selection is based on suppression of amber (TAG) codons in essential or toxic genes, and it has previously been used to evolve aaRS mutants specific for a variety of unnatural amino acids $^{15,18,19}$. We tested the ability of the isolated mutant LeuRS BH5 (M40G, L41Q, Y499L, Y527G, H537F) to selectively incorporate 1 into proteins by suppression of an amber mutant at a permissive site ( $\operatorname{Trp} 33 \rightarrow$ TAG) in $\mathrm{His}_{6}$-tagged human superoxide dismutase (hSOD) (hSOD-33TAG-His 6 ). Subsequent SDS-PAGE analysis indicated that substantial amounts of the TAG mutant were produced in the presence of the unnatural amino acid; however, some hSOD33TAG-His 6 was also expressed in the absence of 1 (Supplementary Fig. 2 online). Analysis of the mutant protein by ESI-MS indicated that leucine or isoleucine was also incorporated at position 33 (Supplementary Fig. 3 online). In order to increase the fidelity of the mutant LeuRS BH5, we mutated residue Thr252 of the editing domain to alanine (LeuRS BH5 T252A). According to previously published work, this mutation results in increased hydrolysis of LeuRS aminoacylated with leucine and therefore increased fidelity ${ }^{20,21}$. Analysis of mutant hSOD-33TAG-His 6 generated with LeuRS BH5 T252A by gel electrophoresis and ESI-MS revealed that the T252A mutation results in a marked increase in fidelity $(>95 \%)$ in the incorporation of 1 , with a yield of $0.8 \mathrm{mg} \mathrm{hSOD}$ mutant protein from a one-liter culture (Fig. $\mathbf{1 b}$ and Supplementary Fig. 3).

To demonstrate the utility of $\mathbf{1}$ in cellular studies, we used this amino acid to photoregulate phosphorylation of Pho4 (Fig. 1c), a transcription factor that is central to the signaling cascade that allows sive genes ${ }^{1}$. In the presence of high concentrations of extracellular $\mathrm{P}_{\mathrm{i}}$, Pho4 is phosphorylated by the cyclin-cyclin dependent kinase (cyclinCDK) complex (Pho80-Pho85), which inactivates Pho4 and triggers its translocation to the cytoplasm ${ }^{23}$ (Fig. 2a). Phosphorylation of Ser223 (termed S6) regulates transcription, whereas phosphorylation of Ser114 (S2) and Ser128 (S3) triggers nuclear export of Pho4 by the receptor Msn5 (ref. 2). Before nuclear export, Pho4 can also be phosphorylated at Ser152 (S4), which in turn prevents reimport of Pho 4 into the nucleus. There is a preference for phosphorylation: $\mathrm{S} 6$ (transcription) $>\mathrm{S} 4$ (import) $>\mathrm{S} 2 / \mathrm{S} 3$ (export region) $>\mathrm{S} 1$ (ref. 24). Moreover, phosphorylation of Pho4 only at the S6 site It has also been suggested that differential gene expression could be regulated by phosphorylation at the export region ( $\mathrm{S} 2$ and $\mathrm{S} 3$ site $)^{25}$, but no mechanism is known and it is not clear whether differential phosphorylation of S2 and S3 could lead to distinct responses in Pho4 activity.

The selective introduction of $\mathbf{1}$ at specific sites in the export region should allow one to dissect the effects of specific phosphorylation of each of these sites on the dynamics of protein trafficking between the nucleus and cytoplasm. To this end, we first substituted a nonsense amber mutation for the $\mathrm{S} 3$ codon $(\mathrm{S} 3 \rightarrow \mathrm{TAG}$ ) in a wild-type (WT) Pho4-GFP (green fluorescent protein) fusion construct and coexpressed the mutated gene with the mutant suppressor tRNA $\mathrm{CUA}_{\mathrm{CU}}^{\mathrm{Leu}} /$ LeuRS pair in $S$. cerevisiae in presence of 1 to generate the $S 3 \rightarrow \mathbf{1}$ Pho4-GFP mutant. Because the phosphorylation site S3 is blocked, the concentrations, which is consistent with the published effect of the $\mathrm{S} 3 \rightarrow$ Ala mutation on localization of Pho4-GFP fusion constructs (Fig. 2) ${ }^{23}$. This demonstrates that the S3 $\rightarrow 1$ Pho4-GFP is still recognized by the import receptor Pse1 (ref. 26) and localized to the nucleus when the S3 phosphorylation site is blocked (additionally, the photocaged Pho4-GFP can also be transcriptionally active-see Supplementary Fig. 4 online). Cells grown in high $\mathrm{P}_{\mathrm{i}}$ in the absence of 1 showed no significant fluorescence (other than autofluorescence, Fig. 2c), which indicates that there is little background translational read-through to the C-terminal GFP. can lead to transcription of a subset of phosphate-responsive genes ${ }^{25}$. $\mathrm{S} 3 \rightarrow 1$ Pho4-GFP mutant accumulates in the nucleus even at high $\mathrm{P}_{\mathrm{i}}$ 
Figure 3 Real-time analysis of in vivo photolysis of DMNB-Ser Pho4-GFP mutants. (a) The normalized average fluorescence intensity is plotted as a function of time for S3 $\rightarrow 1$ Pho4-GFP (gray: photolyzed, filled squares, number of nuclei $n=71$; nonphotolyzed, open circles, $n=67$ ) and S2 $\rightarrow 1$ Pho4-GFP (black: photolyzed, filled squares, $n=101$; nonphotolyzed, open circles, $n=101$ ). The solid lines depict monoexponential fits with time constants of $\tau_{\mathrm{S} 3 \mathrm{TAG}}=28.4 \pm 2.4 \mathrm{~s}$ (gray) and $\tau_{\mathrm{S} 2 \mathrm{TAG}}=47.0 \pm 5.1 \mathrm{~s}$ (black). $(b, c)$ Nuclear fluorescence is still visible after photolysis of the S2 $\rightarrow 1 \mathrm{~S} 3 \rightarrow$ Ala Pho4-GFP (b) and S3 $\rightarrow 1$ S2 $\rightarrow$ Ala Pho4-GFP (c) mutants. (d) Analysis for the S3 $\rightarrow 1$ S2 $\rightarrow$ Ala Pho4-GFP mutant cells in high $\mathrm{P}_{\mathrm{i}}$ (red: photolyzed, filled squares connected by red line, $n=109$ ) and no $\mathrm{P}_{\mathrm{i}}$ (orange: photolyzed, filled squares $n=156$ ), and for the S2 $\rightarrow 1$ S3 $\rightarrow$ Ala Pho4-GFP mutants in high $\mathrm{P}_{\mathrm{i}}$ (purple: photolyzed, filled squares $n=80$; nonphotolyzed, open circles $n=60$, for better clarity only this baseline is shown) and no $P_{i}$ (light blue: photolyzed, filled squares $n=123$ ). Error bars show s.e.m.; ${ }^{* \star} P<0.01$.

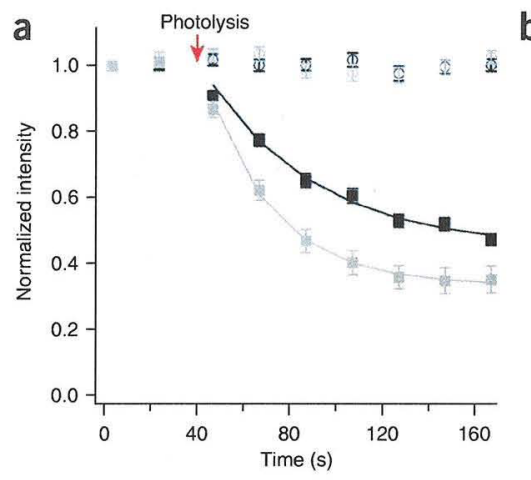

b Before photolysis
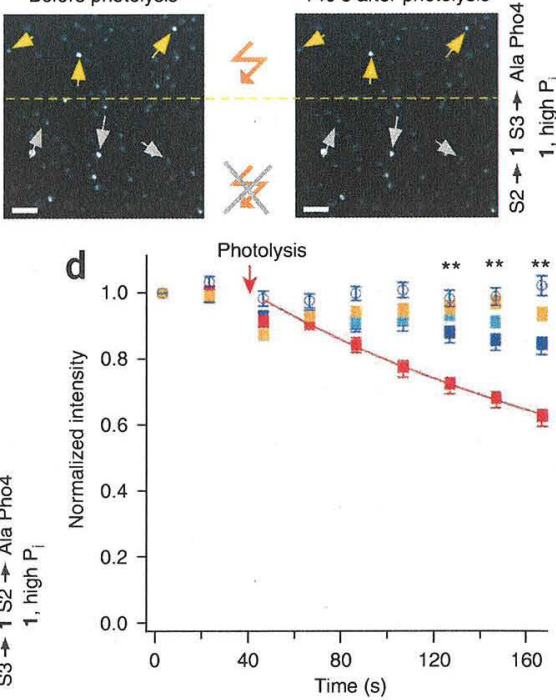

Laser photolysis of the photocaged S3 $\rightarrow 1$ Pho4-GFP mutant should result in photodeprotection of 1 to afford WT Pho4-GFP, which can then be phosphorylated by the cyclin-CDK complex and exported from the nucleus into the cytoplasm. To test this notion, we photolyzed cells expressing $\mathrm{S} 3 \rightarrow 1$ Pho4-GFP in the presence of high $\mathrm{P}_{\mathrm{i}}$ by scanning only the upper part of the microscope field with a visible blue light laser ( $\lambda=405 \mathrm{~nm}$, Fig. 2d). The lower part of each field was not irradiated and therefore served as an internal control in all experiments. We acquired a time series before and after photolysis using excitation at $\lambda=488 \mathrm{~nm}$, which is beyond the absorption wavelength of the caging group (Supplementary Fig. 1). Fluorescence from nuclei in the photolyzed area of the image disappeared over time (Fig. 2d). In addition, Figure $2 \mathrm{e}$ shows two exemplary cells before and after photolysis and a corresponding line scan that shows the decrease in nuclear fluorescence and a small increase in fluorescence in the larger cytoplasmic volume after photolysis. These results demonstrate that the function of WT Pho4-GFP can be restored by removal of the photolabile protecting group with a short visible light trigger, thus resulting in export from the nucleus by Msn5 in the presence of extracellular $\mathrm{P}_{\mathrm{i}}$.

To study the kinetics of Pho4 translocation, we analyzed the acquired image series of the S3 $\rightarrow 1$ Pho4-GFP mutant as a function of time before and after photolysis (Fig. 3a). Because of the low contrast in the cytoplasmic region, only fluorescence changes in the nucleus were measured in every image and analyzed over time. We used a fully automated procedure for data analysis that is based on a wavelet transformation ${ }^{27}$ to detect fluorescent nuclei (Supplementary Fig. 5 online). The photolyzed nuclei showed a fluorescence decrease that followed a time constant of $\tau_{\mathrm{S} 3 \mathrm{TAG}}=28.4 \pm 2.4 \mathrm{~s}$ (errors for time constants depict jackknife estimates ${ }^{28}$, see Supplementary Fig. 5 for details on data processing and data fitting). This time constant is consistent with previous experiments that found the translocation process to be complete within 3-6 min after addition of phosphate to $\mathrm{P}_{\mathrm{i}}$-starved cells ${ }^{2}$.

To identify possible differences between phosphorylation at the S2 and S3 sites, we generated three additional mutants, including singlepoint DMNB-Ser mutants and mutants in which the other phosphorylation site was converted to alanine. We repeated the uncaging experiments with a S2 $\rightarrow \mathbf{1}$ Pho4-GFP mutant (Fig. 2f shows a fluorescence loss similar to that shown in Fig. 2d) and measured slower export kinetics $\left(\tau_{\mathrm{S} 2 \mathrm{TAG}}=47.0 \pm 5.1 \mathrm{~s}\right.$, Fig. $\left.3 \mathrm{a}\right)$, which indicates that the $\mathrm{S} 2 \rightarrow 1$ and $\mathrm{S} 3 \rightarrow 1$ Pho4-GFP mutants have differential phosphorylation or export kinetics.

We then analyzed the S2 $\rightarrow 1$ S3 $\rightarrow$ Ala Pho4-GFP and S3 $\rightarrow 1$ S2 $\rightarrow$ Ala Pho4-GFP double mutants (Fig. 3b,c). Unlike the single mutants studied above, these mutants can only be phosphorylated at one site of the export region. In agreement with the previously reported effect of S3 $\rightarrow$ Ala Pho4 mutants ${ }^{23}$, we found that nuclear export remained blocked after photolysis $\left(\tau_{S_{2} T_{A G} S 3 A_{a}} \sim 0\right.$, Fig. $\left.3 d\right)$. In contrast, there was still a slow kinetic decay for the S3 $\rightarrow 1$ S2 $\rightarrow$ Ala Pho4-GFP mutant $\left(\tau_{\text {S3TAG S2Ala }}>100 \mathrm{~s}\right)$. This decay is absent if cells are additionally starved for $\mathrm{P}_{\mathrm{i}}$, which indicates that it arises from residual export activity (Fig. 3d). Previous measurements of S3 $\rightarrow$ Ala Pho4GFP localization under steady state conditions did not reveal this activity, possibly because of competing import activity. Taken together, our data suggest that differential phosphorylation at sites S2 and S3 leads to distinct export kinetics for these isoforms of Pho4. It is possible that this differential activity is part of the mechanism regulating differential gene expression, by controlling the kinetic localization of Pho4 in the nucleus.

In summary, we described a method to nondestructively control protein function in vivo with high spatial and temporal resolution. We used this methodology to photoinitiate the phosphorylation of Pho4 by the cyclin-CDK complex and to monitor the kinetics of protein trafficking in real time. This general method should be applicable to other proteins in which serine is involved in a specific binding interaction or catalytic activity, and it is currently being extended to additional residues and to mammalian cells.

\section{METHODS}

Evolution of tRNA synthetases for incorporation of 1 . To evolve a LeuRS specific for 1 , a previously published protocol was used ${ }^{18}$. Briefly, positive selection was carried out by suppression of two amber (TAG) codons in the gene encoding the transcriptional activator GAL4. Production of functional full-length GAL4 drives expression of a genomic URA3 reporter gene and complements uracil auxotrophy, thus allowing clones harboring active synthetase mutants to be selected on synthetic dropout medium lacking uracil (SD 
medium containing dextrose) supplemented with $1 \mathrm{mM} 1$ (stock solutions of 1 are $0.1 \mathrm{M}$ in $0.1 \mathrm{M} \mathrm{HCl}$ ). Negative selection of synthetases that accept endogenous amino acids was carried out by growth on medium lacking 1 but containing $0.1 \% 5$-fluoroorotic acid, which is converted into a toxic product by URA3 protein. Based on its growth rate in the presence and absence of $\mathbf{1}$, clone BH5 emerged after five alternating rounds of positive and negative selection. The T252A mutation was introduced into the plasmid pescTrpLeuRSBH5 isolated from the selected clone BH5 as described previously ${ }^{18}$ to afford plasmid pescTrpLeuRSBH5T252A.

Expression, purification and characterization of hSOD-His ${ }_{6}$ harboring 1. Protein expression was performed with strain SCY4 (MATa, ade2-101 ura3-1 leu2-3, 112 trp1 his3-11 $\triangle C Y B 2:: k a n$ [cir0]) transformed with the hSOD expression plasmid pC1SOD-33TAG-His 6 (ref. 18) and the tRNA $\mathrm{CUA}_{\mathrm{CUA}}^{\mathrm{Lew}} / \mathrm{LeuRS}$ encoding plasmid (pescTrpLeuRS derivatives). Cultures were grown to saturation at $30{ }^{\circ} \mathrm{C}$ in SD medium lacking leucine and tryptophan and diluted 50 -fold into SD medium (containing dextrose) lacking leucine and tryptophan and containing $4 \mathrm{mM} 1$. This culture was incubated for $16 \mathrm{~h}$ at $30^{\circ} \mathrm{C}$ and cells were harvested. Lysis and purification was performed as described previously, except that washing buffer contained $50 \mathrm{mM}$ instead of $25 \mathrm{mM}$ imidazole ${ }^{18}$. SDS-PAGE analysis was performed using GelCode Blue Stain (Pierce). Yields were routinely quantified using a BCA assay (Pierce).

Cloning and expression of DMNB-Ser Pho4-GFP mutants. Pho4-GFP (ref. 1) was a gift (see Acknowledgments). Mutants were generated using the QuickChange method to introduce either TAG or alanine mutations at Ser114 (S2) and/or Ser128 (S3). The respective Pho4-GFP mutant genes were inserted into the pesc-His vector (Stratagene) using NotI and Sacl sites and expressed under control of the Gall inducible promotor. The plasmid was cotransformed into an INVSC1 strain (Invitrogen) harboring plasmid pescTrpLeuRSBH5T252A. The strain was grown in SD medium lacking galactose and 1 until mid-log phase, pelleted and resuspended in medium containing $2 \%$ galactose and $2 \%$ raffinose at a similar optical density. $4 \mathrm{mM} 1$ was then added to this medium. Cells were allowed to grow in the dark for an additional 7-8 h, washed and resuspended in yeast nitrogen base (Formedium; plus $2 \%$ glucose) and $20 \mathrm{mM}$ $\mathrm{KH}_{2} \mathrm{PO}_{4}$ (for high $\mathrm{P}_{\mathrm{i}}$ measurement). For measurements in the absence of $\mathrm{P}_{\mathrm{i}}$, cells were allowed to grow for 7-8 h, washed and resuspended in two volumes of yeast nitrogen base plus $2 \%$ glucose plus $20 \mathrm{mM} \mathrm{KCl}$. Cells were grown for an additional $2-3 \mathrm{~h}$ before the imaging experiment.

Real-time imaging and analysis. Experiments were performed on an inverted Bio-Rad (Zeiss) Radiance 2100 Rainbow laser scanning confocal microscope with a 1.4 numerical aperture oil objective and an $\operatorname{argon}$ laser $(\lambda=488 \mathrm{~nm})$. For photolysis a visible blue light laser $(\lambda=405 \mathrm{~nm})$ was used. The scan speed was adjusted to 166 lines per second, resulting in a pixel dwell time of about $10.4 \mu \mathrm{s}$. The image size was 512 by 512 pixels $(82 \mu \mathrm{m}$ by $82 \mu \mathrm{m})$, with a pixel size of $0.16 \mu \mathrm{m}$ by $0.16 \mu \mathrm{m}$. Thus, for a typical nucleus with a size of 10 by 10 pixels, the total laser light exposure time was approximately $1 \mathrm{~ms}$. The image depth was 8 bit, and displayed images were filtered with a 3 by 3 median filter. The laser power measured at the nose piece of the objective was approximately $65 \mu \mathrm{W}$ at $\lambda=488 \mathrm{~nm}$ and $410 \mu \mathrm{W}$ at $\lambda=405 \mathrm{~nm}$ in all experiments. For quantitative analysis of the real-time data, the automated procedure described in Supplementary Figure 5 was used.

Statistical analysis. Statistical analysis in Figure 3d was done using a one-way ANOVA to perform a Dunnett's test between photolysed cells harboring S3TAG $\rightarrow 1$ S2 $\rightarrow$ Ala Pho4-GFP grown in the presence of high $P_{i}$ and the other data shown in this panel for each time point. Significance levels were assigned to $P$ values according to ${ }^{*} P<0.05$ and ${ }^{* *} P<0.01$. All analyses were performed using Igor Pro (Wavemetrics). Error bars are given as s.e.m.

\section{ACKNOWLEDGMENTS}

We thank E. O'Shea (Howard Hughes Medical Institute, Harvard University) for the PRS-Pho4 ${ }^{\text {WT }}$-GFP plasmid, the mass spectrometry facility of the Genomics Institute of the Novartis Research Foundation for protein mass measurements and E. Peters for helpful discussions. E.A.L. and D.S. acknowledge a postdoctoral scholarship from the Alexander von Humboldt Foundation. This work was also supported by the US Department of Energy (DE-FG03-00ER46051) and the Skaggs Institute for Chemical Biology.

\section{AUTHOR CONTRIBUTIONS}

E.A.L. designed experiments, performed biological experiments, imaging and data analysis, and wrote the manuscript; D.S. designed experiments, evolved the synthetase, performed biological experiments and wrote the manuscript; B.H.G. and S.M.B. provided mass spectrometric analysis; B.H.G. assisted in editing the manuscript; P.G.S. designed experiments and wrote the manuscript.

1. O'Neill, E.M., Kaffman, A., Jolly, E.R. \& O'Shea, E.K. Regulation of PHO4 nuclear loca lization by the PHO80-PHO85 cyclin-CDK complex. Science 271, 209-212 (1996). 2. Kaffman, A., Rank, N.M., O'Neill, E.M., Huang, L.S. \& O'Shea, E.K. The receptor Msn5 exports the phosphorylated transcription factor Pho4 out of the nucleus. Nature 396, 482-486 (1998).

3. Pelliccioli, A.P. \& Wirz, J. Photoremovable protecting groups: reaction mechanisms and applications. Photochem. Photobiol. Sci. 1, 441-458 (2002).

4. Mayer, G. \& Heckel, A. Biologically active molecules with a "light switch". Angew. Chem. Int. Edn Engl. 45, 4900-4921 (2006).

5. Lawrence, D.S. The preparation and in vivo applications of caged peptides and proteins. Curr. Opin. Chem. Biol. 9, 570-575 (2005).

6. Rothman, D.M., Shults, M.D. \& Imperiali, B. Chemical approaches for investigating phosphorylation in signal transduction networks. Trends Cell Biol. 15, 502-510 (2005).

7. Ghosh, M., Ichetovkin, I., Song, X., Condeelis, J.S. \& Lawrence, D.S. A new strategy for caging proteins regulated by kinases. J. Am. Chem. Soc. 124, 2440-2441 (2002).

8. Zou, K., Cheley, S., Givens, R.S. \& Bayley, H. Catalytic subunit of protein kinase A caged at the activating phosphothreonine. J. Am. Chem. Soc. 124, 8220-8229 (2002).

9. Endo, M., Nakayama, K., Kaida, Y. \& Majima, T. Design and synthesis of photochemically controllable caspase-3. Angew. Chem. Int. Edn. Engl. 43, 5643-5645 (2004).

10. Hahn, M.E. \& Muir, T.W. Photocontrol of Smad2, a multiphosphorylated cell-signaling protein, through caging of activating phosphoserines. Angew. Chem. Int. Edn. Engl. 43, 5800-5803 (2004).

11. England, P.M., Lester, H.A., Davidson, N. \& Dougherty, D.A. Site-specific, photochemical proteolysis applied to ion channels in vivo. Proc. Natl. Acad. Sci. USA 94 11025-11030 (1997).

12. Volgraf, M. et al. Allosteric control of an ionotropic glutamate receptor with an optical switch. Nat. Chem. Biol. 2, 47-52 (2006).

13. Banghart, M., Borges, K., Isacoff, E., Trauner, D. \& Kramer, R.H. Light-activated ion channels for remote control of neuronal firing. Nat. Neurosci. 7, 1381-1386 (2004)

14. Deiters, A., Groff, D., Ryu, Y., Xie, J. \& Schultz, P.G. A genetically encoded photocaged tyrosine. Angew. Chem. Int. Edn Engl. 45, 2728-2731 (2006)

15. Wu, N., Deiters, A., Cropp, T.A., King, D. \& Schultz, P.G. A genetically encoded photocaged amino acid. J. Am. Chem. Soc. 126, 14306-14307 (2004).

16. Walker, J.W., Martin, H., Schmitt, F.R. \& Barsotti, R.J. Rapid release of an alphaadrenergic receptor ligand from photolabile analogues. Biochemistry 32, 1338-1345 (1993)

17. Cusack, S., Yaremchuk, A. \& Tukalo, M. The 2 A crystal structure of leucyl-tRNA synthetase and its complex with a leucyl-adenylate analogue. EMBO J. 19 , 2351-2361 (2000).

18. Summerer, D. et al. A genetically encoded fluorescent amino acid. Proc. Natl. Acad. Sci. USA 103, 9785-9789 (2006).

19. Xie, J. \& Schultz, P.G. A chemical toolkit for proteins-an expanded genetic code. Nat Rev. Mol. Cell Biol. 7, 775-782 (2006).

20. Mursinna, R.S. \& Martinis, S.A. Rational design to block amino acid editing of a tRNA synthetase. J. Am. Chem. Soc. 124, 7286-7287 (2002).

21. Lincecum, T.L., Jr. et al. Structural and mechanistic basis of pre- and posttransfer editing by leucyl-tRNA synthetase. Mol. Cell 11, 951-963 (2003).

22. Oshima, Y. The phosphatase system in Saccharomyces cerevisiae. Genes Genet. Syst 72, 323-334 (1997)

23. Komeili, A. \& O'Shea, E.K. Roles of phosphorylation sites in regulating activity of the transcription factor Pho4. Science 284, 977-980 (1999).

24. Jeffery, D.A., Springer, M., King, D.S. \& O'Shea, E.K. Multi-site phosphorylation of Pho4 by the cyclin-CDK Pho80-Pho85 is semi-processive with site preference. J. Mol. Biol. 306, 997-1010 (2001).

25. Springer, M., Wykoff, D.D., Miller, N. \& O'Shea, E.K. Partially phosphorylated Pho 4 activates transcription of a subset of phosphate-responsive genes. PLOS Biol. 1, E28 (2003).

26. Kaffman, A., Rank, N.M. \& O'Shea, E.K. Phosphorylation regulates association of the transcription factor Pho4 with its import receptor Pse1/Kap121. Genes Dev. 12 2673-2683 (1998).

27. Olivo-Marin, J.C. Extraction of spots in biological images using multiscale products Pattern Recognit. 35, 1989-1996 (2002).

28. Thomson, D.J. \& Chave, A.D. in Advances in Spectrum Estimation (ed. Haykin, S.) 58-113 (Prentice Hall, Englewood Cliffs, New Jersey, USA, 1991).

29. Veldhuyzen, W.F., Nguyen, Q., McMaster, G. \& Lawrence, D.S.A. Light-activated probe of intracellular protein kinase activity. J. Am. Chem. Soc. 125, 13358-13359 (2003). 